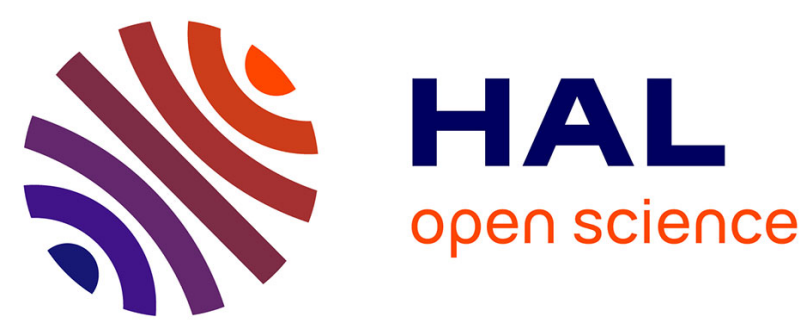

\title{
Distributed X-Ray Dosimetry With Optical Fibers by Optical Frequency Domain Interferometry
}

\author{
Massimo Olivero, Alessandro Mirigaldi, Valentina Serafini, Alberto Vallan, \\ Guido Perrone, Wilfried Blanc, Mourad Benabdesselam, Franck Mady, Carlo \\ Molardi, Daniele Tosi
}

\section{To cite this version:}

Massimo Olivero, Alessandro Mirigaldi, Valentina Serafini, Alberto Vallan, Guido Perrone, et al.. Distributed X-Ray Dosimetry With Optical Fibers by Optical Frequency Domain Interferometry. IEEE Transactions on Instrumentation and Measurement, 2021, 70, pp.1-9. 10.1109/TIM.2021.3075518 . hal-03441945

\section{HAL Id: hal-03441945 \\ https://hal.science/hal-03441945}

Submitted on 22 Nov 2021

HAL is a multi-disciplinary open access archive for the deposit and dissemination of scientific research documents, whether they are published or not. The documents may come from teaching and research institutions in France or abroad, or from public or private research centers.
L'archive ouverte pluridisciplinaire HAL, est destinée au dépôt et à la diffusion de documents scientifiques de niveau recherche, publiés ou non, émanant des établissements d'enseignement et de recherche français ou étrangers, des laboratoires publics ou privés. 


\title{
Distributed X-Ray Dosimetry With Optical Fibers by Optical Frequency Domain Interferometry
}

\author{
Massimo Olivero $^{\circledR}$, Alessandro Mirigaldi ${ }^{\circledR}$, Valentina Serafini ${ }^{\circledR}$, Alberto Vallan ${ }^{\circledR}$, Senior Member, IEEE, \\ Guido Perrone $^{\circledR}$, Member, IEEE, Wilfried Blanc ${ }^{\circledR}$, Mourad Benabdesselam ${ }^{\circledR}$, Franck Mady ${ }^{\circledR}$, \\ Carlo Molardi $^{\circledR}$, Member, IEEE, and Daniele Tosi ${ }^{\circledR}$
}

\begin{abstract}
This article reports on the first demonstration of in situ, real-time dosimetry realized with an enhanced backscattering optical fiber, and a high-resolution optical backscattering reflectometry measurement. This work is devised to overcome the current problems in monitoring radiotherapy treatments, in particular, the difficult evaluation of not only the actual $\mathrm{X}$-ray dose that is accumulated on the target volume but also the distribution profile of the ionizing radiation beam. Overall, the research aims at developing a dose sensor with the most demanding features of small form factor, spatial profiling, and remote interrogation. The experiments have been conducted by evaluating the spatial profile of radiation-induced spectral shift of the Rayleigh backscattering along an optical fiber exposed to X-rays. The sensing element is a section of specialty optical fiber whose Rayleigh backscattering signature changes under ionizing radiation. The specialty fiber is designed to exhibit an enhanced backscattering, in order to overcome the poor sensitivity to radiation of standard optical fibers that are normally, used in telecommunications. The enhanced sensitivity is achieved by doping the core with either aluminum or magnesium nanoparticles, and two different fibers have been fabricated and tested. The experimental results show the capability of real time detection of the radiation profile from high-dose rates ( $700 \mathrm{~Gy} / \mathrm{min}$ ) to low-dose rates $(2 \mathrm{~Gy} / \mathrm{min})$. Moreover, different sensing mechanisms and responses to high- and low-dose rates are evidenced. A comparison with a quasi-distributed sensing system based on an array of fiber Bragg gratings (FBGs) is discussed, highlighting the superior performance of the backscattering approach in terms of sensitivity and spatial resolution, whereas the array of FBGs exhibits an advantage in terms of sampling speed.
\end{abstract}

Index Terms-Ionizing radiation sensors, optical fiber sensors, radiation dosage, radiation monitoring.

\section{INTRODUCTION}

I ONIZING radiations find many medical applications, going from diagnostic, such as computed tomography (CT), to therapeutics, such as tumor radiotherapy. These two fields are characterized by quite different dose levels, practically at the opposite of the scale: extremely low doses for imaging to investigate tissues without producing damages and relatively high doses for radiotherapy to kill cancer cells or slow their growth by damaging their DNA. In both cases, however, modern medical protocols require accurate monitoring of the dose at which the patient is exposed. While this can be straightforward to understand for the high doses used in therapeutic applications, it has to be pointed out that low doses used in imaging equipment can be harmful too; for example, if, for some reason, the instrument stops working properly: the literature reports cases of patients who have filed lawsuits for having suffered burns from extreme overexposures during CT exams due to improper settings on the CT scanners.

Ionizing radiations can be measured using a number of instruments, the most common being personal dosimeters in the form of film badges worn on clothing, which measures the cumulative radiation dose a person receives while exposed to radiation sources. More quantitative evaluations can be made with hand-held detectors, such as Geiger-Muller counters and ionization chambers, which are used to measure the exposure rate from a source (e.g., emissions from radioactive wastes). In medical applications, however, it is interesting not only to measure the total dose at which the patient is exposed but also the spatial distribution of the radiation in the target volume. Unfortunately, the latter measurement is difficult to implement in real time before and during the treatment. Indeed, the previously mentioned radiation monitoring instruments cannot be used because they measure the amount of radiation on a quite large area and, thus, are not easy to be multiplexed to recover the radiation distribution, not to mention the poor resolution achievable in that case. The most common technique to evaluate the intensity distribution is to use GafChromic ( $\mathrm{GaF})$ films. These films contain a special dye that polymerizes upon exposure to radiation, with a subsequent increase in its absorption at optical frequencies, that is, the optical density (OD) of the film increases with the dose [1], turning it more opaque (see Fig. 1).

Another alternative proposed in the literature exploits gelbased nanosensors, in which exposure to ionizing radiation results in the conversion of gold ions to nanoparticles, with subsequent visual change in color due to their plasmonic properties [2]. Both approaches, however, cannot be used for real-time continuous measurements [3]. 


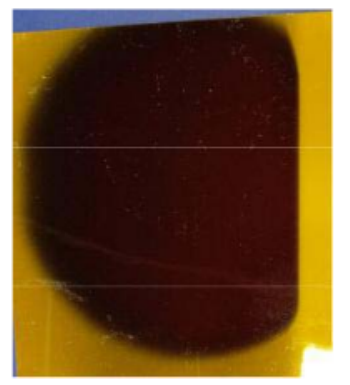

Fig. 1. GaF film exposed at $700 \mathrm{~Gy} / \mathrm{min}$. The spot size is about $20 \mathrm{~mm}$.

Optical fibers can constitute an attractive alternative technology for the realization of a new class of ionizing radiation sensors due to a number of unique features, among which: 1) they are minimally invasive; 2) they can be remotely interrogated; and 3) they do not involve electrical currents (a plus feature in medical applications as it intrinsically avoids electrocution). Standard optical fibers for telecommunication applications are made by ultrapure silica, with some dopants added to slightly increase the refractive index in the core region (usually, germanium or phosphorus), and exhibit little sensitivity to ionizing radiations; however, it is known from the literature that this low sensitivity can be enhanced by codoping with aluminum $(\mathrm{Al})$.

The sensitivity of fibers to radiation typically manifests in the form of radiation-induced attenuation (RIA), for which the propagation loss of an irradiated fiber is higher than that of the pristine fiber. This is a local effect, so the loss increase against the fiber length is proportional to the cumulative radiation along with the fiber. In other words, this effect can be exploited to evaluate the radiation intensity profile by measuring the loss spatial distribution along with the fiber, a kind of measurement that, in telecommunication applications, is performed with an optical time-domain reflectometer (OTDR). In the OTDRs, a light pulse of known width is transmitted through the fiber, and the reflected power and the time of flight are measured to determine the magnitude and location of the perturbations along with the fiber. An application of OTDRs for long-range gamma-ray detection has been presented in [4]. Furthermore, the OTDR technique has been used to qualify the RIA of different optical fibers, by exposing samples with lengths of about $10 \mathrm{~m}$ [5]. However, besides having a dead zone (i.e., an initial length in which the interrogator is blind), in the order of meters, OTDRs are nonsuitable for radiotherapy dosimetry applications for the poor resolution, in terms of both loss and position, especially considering that, for this application, the radiation intensity (and, thus, the loss variation) is expected to be small, and the dose profile should ideally be measured with subcentimeter resolution. Therefore, another radiation-dependent feature must be investigated. When an optical signal propagates along with a fiber, a small amount of its power is backscattered because of a phenomenon known as the Rayleigh scattering, which depends on the local composition fluctuation in a subwavelength scale. The associated attenuation is usually low in commercial fibers and, as previously stated, quite insensitive to radiation. On the other hand, the real part of the refractive index, responsible for the phase delay

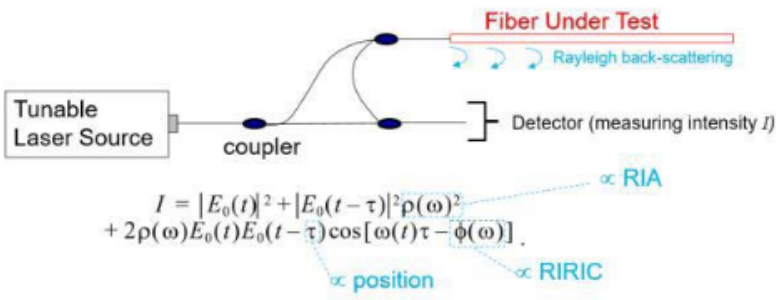

Fig. 2. Schematic representation of the OFDR principle of operation. The intensity $I$ measured by the detector contains information about the RIRIC and the RIA along with the fiber under test. $j E_{0}(t) j^{2}$ is the intensity provided by the tunable laser; (!) is the spectral response of the device (in this case, a fiber) under test; and ! is related to the current wavelength of the swept laser and $t$, and the time and time delay of the observation. By performing autocorrelation and subsequent Fourier transform of $I$, it is possible to evaluate RIA and RIRIC along with the fiber.

of the signal propagating along with the fiber, can be more effectively correlated with ionizing radiation. In particular, the Rayleigh backscattering can be measured with high accuracy through optical frequency-domain reflectometry (OFDR). The principle of operation of OFDR may be better explained with the help of the schematic in Fig. 2. OFDR instruments rely on a swept wavelength laser coupled into a modified Mach-Zender interferometer. One leg of the interferometer is a reference path of fixed length, while the other leg is the optical fiber under test interrogated in reflection through a directional coupler. The backscattered light is combined with the light from the reference arm generating an interference signal that contains information related to the accurate location (i.e., with submillimeter resolution) and magnitude of reflective events along the fiber length. In fact, an acquisition system collects a large number of intensity signals from the detector, as a function of wavelength and time (by performing multiple sweeps). The intensity patterns are cross-correlated, and from the cross correlation function (or, better, its Fourier transform), it is possible to retrieve the complete spectral response (both in terms of real and imaginary parts) at any position along with the fiber under test. Thus, the OFDR is able to measure the precise location of very small reflective events with no dead zone.

A recent review article, with details on the principle of operation and a comprehensive overview of its sensing applications, can be found in [6].

In this work, enhanced backscattering fibers (EBFs) were exposed to X-rays, while their Rayleigh scattering was recorded using an OFDR. Then, the Rayleigh scattering signature was processed in real time to calculate a spectral shift parameter that could be correlated with the dose, through a linear relationship. The RIA was also measured to prove its weak effect and the need for more advanced measurement techniques, such as the OFDR for the evaluation of the radiation profile. The novelty of this research relies both on exploiting radiation-sensitive custom-made optical fibers and investigating the spectral shift of the Rayleigh backscattering rather than measuring the RIA, by exploiting a high-resolution OFDR interrogation. The results are expected to pave the way to the development of novel distributed dosimeters based on optical fibers. The work here reported is an extension 


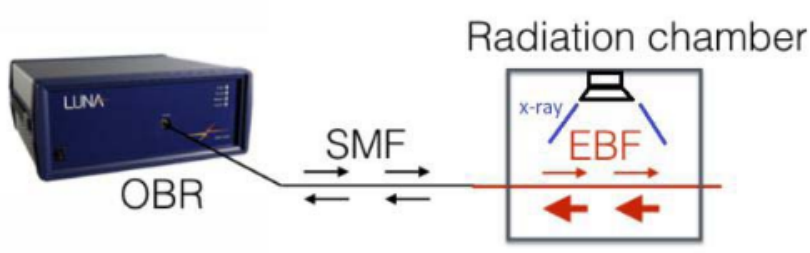

Fig. 3. Schematic representation of the measurement setup.

of that presented at the 15th edition of the IEEE International Symposium on Medical Measurements and Applications (MeMeA 2020) [7], and it reports a remarkable amount of new analyses, extending the scope of the former article to the comparison of two different EBFs while reporting a considerable improvement in data acquisition reliability. Last but not least, the current work also reports the response to X-ray exposure of femtosecond-written fiber Bragg gratings (FBGs) in one of the EBFs, confirming the capability to measure the radiation-induced refractive index change (RIRIC) along with the irradiated fiber and opening an alternative for quasidistributed radiation profile evaluation.

\section{Measurement Setup}

The experimental setup is sketched in Fig. 3, and it is built around an interrogation unit constituted by a Luna OBR4600 optical backscatter reflectometer (OBR) [8]. The OBR is a swept-laser interferometer that implements an OFDR principle. The system is capable of sensing Rayleigh scattering signatures along with the fiber under test, with a nominal spatial resolution of $<10 \mu \mathrm{m}$, operating on an 88-nm wavelength grid centered around the infrared $C$-band $(1550 \mathrm{~nm})$ [9], [10]. The fiber to be tested as a dosimeter is positioned in a shielded radiation chamber, under an X-ray beam, and connected to the OBR through an about $4.5 \mathrm{~m}$ standard single-mode fiber (SMF). The X-ray generator (INEL, model XRG3D) is based on a tube containing a copper target with an accelerating voltage of $30 \mathrm{kV}$. The cone-like X-ray emission, produced in air and at room temperature, is controlled by adjusting the supplied electrical current and the distance between the generator and the fiber under test. The beam was calibrated, in terms of dose rate, by means of a flat ionization chamber (PTW, model 23342) and a high-precision/high-resolution electrometer (PTW, model UNIdos E).

Three different irradiation conditions were considered: 1) very high dose by positioning the fiber $26 \mathrm{~mm}$ from the source window, leading to a dose rate of about $710 \mathrm{~Gy} / \mathrm{min}$; 2) medium-high dose obtained by moving the fiber down to $295 \mathrm{~mm}$ from the source window, corresponding to a lower dose rate of about $22 \mathrm{~Gy} / \mathrm{min}$; and 3) low dose of about $2 \mathrm{~Gy} / \mathrm{min}$ resulting from positioning the fiber about $70 \mathrm{~mm}$ from the source.

To overcome the known poor sensitivity to radiation of standard SMFs used in telecommunications (a feature appreciated for that application), the sensing section is an aluminosilicate fiber that exhibits enhanced backscattering. For some of the experiments, a further improved nanoparticle-doped version was used, in which the core is doped with magnesium-based $(\mathrm{Mg})$ nanoparticles, hereby denoted as NPEBF. The fabrication process of these fibers is detailed in several publications, such as in [11] and [14]. It consists of a preform fabrication by a standard modified chemical vapor deposition (MCVD) technique followed by fiber drawing, in which a silicate tube is used to build the preform. The core layers are deposited at a reduced temperature, so as to form unsintered porous soot. The solution doping technique [12] is applied to incorporate $\mathrm{Al}$ or $\mathrm{Mg}$ ions in the core. For this step, the tube is subsequently removed from the lathe and soaked in an alcoholic solution containing the dopant precursor of the desired concentration. After drying the solvent, the tube is replaced in the lathe and heated to produce sintering of the core into a dense glass layer. Finally, it is collapsed at an elevated temperature, higher than $1800{ }^{\circ} \mathrm{C}$, into a solid rod that constitutes the preform for optical fiber drawing. Mg-based nanoparticles in the NPEBF preform are formed due to the phase separation occurring at high temperatures [13]. The preform is pulled at high temperature $\left(>2000{ }^{\circ} \mathrm{C}\right)$ into a drawing tower to produce an optical fiber with a core/cladding diameter of $10 \mu \mathrm{m} / 125 \mu \mathrm{m}$, respectively. SMF and EBF fibers are spliced with a standard fusion splicer, as they have matching core/cladding sizes and similar effective refractive index.

The former experiments presented in the previous MeMeA article were taken by manually recording the Rayleigh signatures at a rate of about 1 acquisition/min, and the exposures lasted $10 \mathrm{~min}$. The setup has been then upgraded by implementing an automatic acquisition system. To do so, the Luna OBR4600 is controlled through a custom Labview program that implements handshaking between the controlling computer and the instrument. This way, the acquisition rate is pushed to the instrument capability, which depends on the measurement parameters. The results presented in the following were obtained by setting a spatial resolution of $0: 1 \mathrm{~mm}$ and a gauge length of $10-30 \mathrm{~mm}$, which allowed for the acquisition of one spatial signature every $30 \mathrm{~s}$. The gauge length represents an equivalent sensor length (or, in other words, the number of points), in which the Rayleigh backscattering parameters are computed. Faster recordings could be possible by setting higher (i.e., poorer) spatial resolutions and/or higher gauge lengths, but it must be pointed out that, in any case, the heavy data processing burden limits the capability of the instruments to acquisitions rates in the order of seconds.

\section{EXPERIMENTAL Results}

\section{A. Assessment of the Enhanced-Backscattering Fiber}

The effects of exposure to X-rays were evaluated in terms of the spectral shift of the Rayleigh scattering signature versus longitudinal position along the EBF. In order to qualitatively understand the rationale of spectral shift, one can consider the exposed fiber as a cascade of densely spaced weak FBGs [15]. These FBGs backscatter a portion of the signal provided by the swept laser of the OBR instruments, thus producing a reference signature of that fiber (the Rayleighscattering signature). Any change in the refractive index or in the pitch of each grating will produce a spectral shift of its Bragg reflection peak; the collection of these shifts represents the spectral shift curve with respect to the pristine 


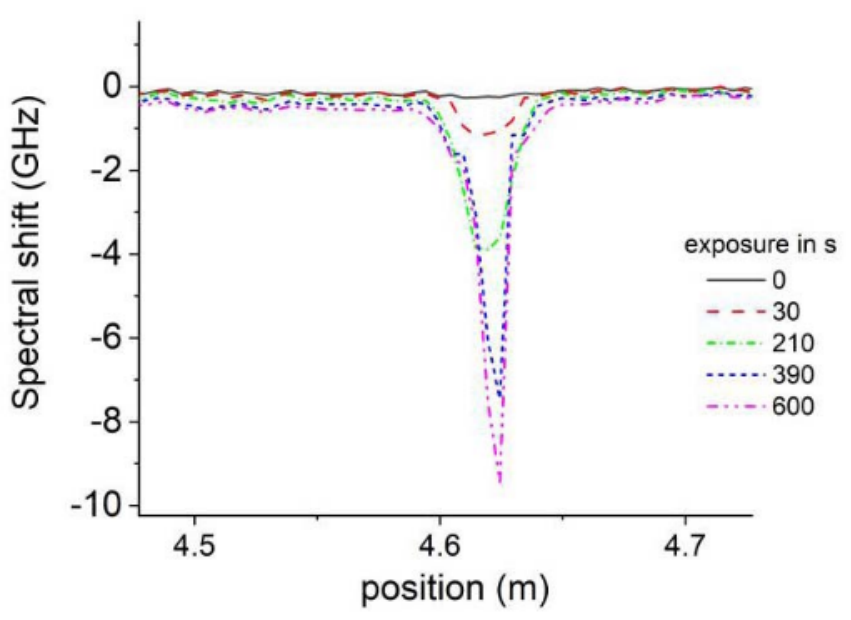

Fig. 4. Spectral shift versus position along the EBF for different times of exposure at $700 \mathrm{~Gy} / \mathrm{min}$. The spatial resolution of the data is $5 \mathrm{~mm}$.

fiber signature. The procedure to measure the spectral shift along the fiber is then performed in a two-steps process: first, the reference signature is acquired in steady conditions; then, the Rayleigh backscattering spectra are subsequently recorded and cross-correlated with the reference to retrieve the spectral shift (these operations are performed by the OBR instrument).

The fiber underexposure was firmly fixed and kept at a constant temperature so that the spectral shift could only be ascribed to the effect of radiation. As already mentioned, the OBR was computer-controlled through a custom-developed program that recorded the spectral shift every $30 \mathrm{~s}$, with a spatial resolution set to $5 \mathrm{~mm}$.

As a preliminary test, a high-dose rate of $700 \mathrm{~Gy} / \mathrm{min}$ was used; the resulting spectral shift is reported in Fig. 4. The graph demonstrates that the considered fiber is sensitive to such a high dose because a clear strong modification of the spectral shift appears exactly where the X-ray exposure occurred. Moreover, the full-width half-minimum (FWHM) of the dip is about $20 \mathrm{~mm}$, in good agreement with a comparative measure performed by $\mathrm{GaF}$ films.

A negative spectral shift is induced, producing a dip that deepens as the energy is deposited on the fiber. Once the X-ray beam is turned off, a partial recovery of the spectral shift is observed, indicating a possible reversible process (permanentinduced defects are likely to occur at higher doses, as observed for example by Girard et al. [16]). The dynamics of the dip at the position 4:62 m, observable in Fig. 5, is reasonably well approximated by a linear dependence on the dose during the exposure. The recovery, instead, exhibits a more complex trend, but, in the graph, it is linearly fitted to highlight the change of sign in the slope and to give a rough estimation of the recovery time, which is about $0.73 \mathrm{GHz} / \mathrm{min}$.

In order to make a quantitative comparison of the sensitivity of the EBF with a standard SMF, the same experiment at the high-dose rate of $700 \mathrm{~Gy} / \mathrm{min}$ was repeated exposing an SMF pigtail (G652 compatible fiber). The SMF exhibited, as expected, a much smaller, yet still detectable, spectral shift located at the exposed section, as shown in Fig. 6.

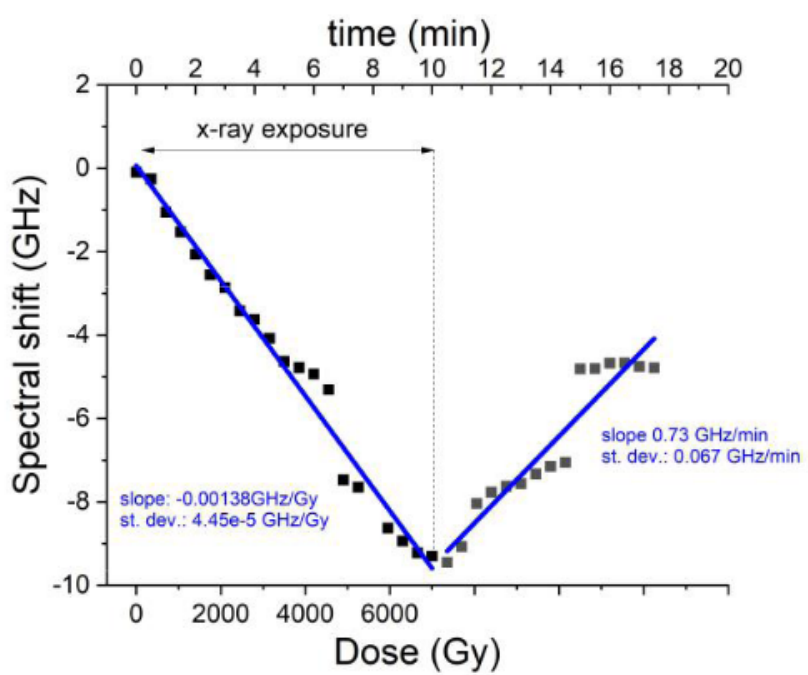

Fig. 5. Spectral shift versus exposure time and corresponding dose for the $\mathrm{EBF}$ at $700 \mathrm{~Gy} / \mathrm{min}$.

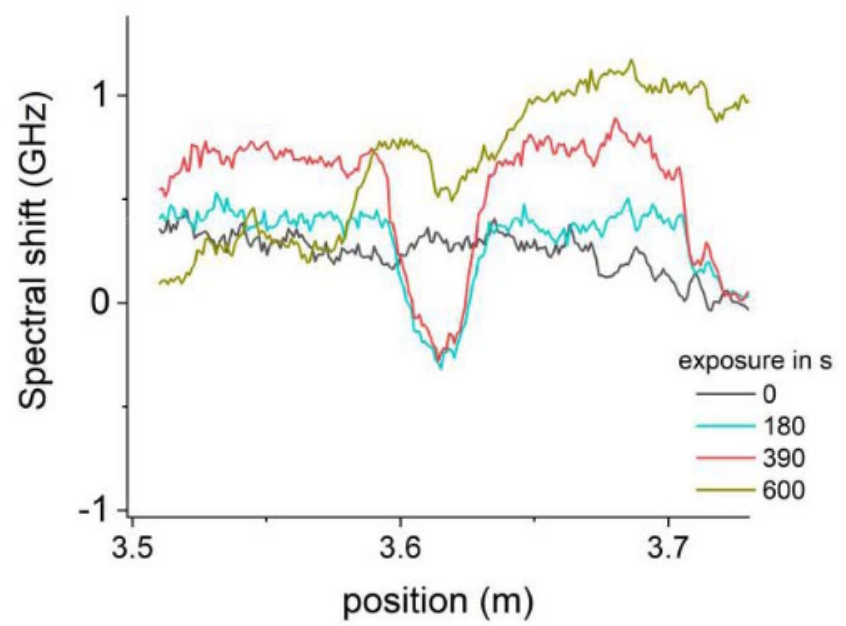

Fig. 6. Spectral shift versus position for a standard SMF (G652 compliant fiber) exposed at $700 \mathrm{~Gy} / \mathrm{min}$.

The final spectral shift for a cumulative dose of $5200 \mathrm{~Gy}$ was about $0: 3 \mathrm{GHz}$ and did not show any further change with time. Fig. 7 reports the spectral shift evolution versus time and corresponding dose. The graph axes are set on the same scales as Fig. 5 to make a straightforward comparison and highlight the different sensitivities between the EBF and the SMF.

The Al-doped fiber was also tested at a low dose of $22 \mathrm{~Gy} / \mathrm{min}$ (see Fig. 8), exhibiting a similar trend for the accumulated dose as for the high-dose exposure (see Fig. 9). In both high-and low-dose experiments, the linear dependence between spectral shift and dose exhibits a slope in the order of magnitude of $0.001-0.002 \mathrm{GHz} / \mathrm{Gy}$. It must be pointed out that the fairly poor reproducibility of the slope coefficient can be ascribed to two reasons: 1) recordings at $22 \mathrm{~Gy} / \mathrm{min}$ were performed with slightly different settings of the OBR to enhance the sensitivity and 2) still, the data from the experiment at $22 \mathrm{~Gy} / \mathrm{min}$ were at the limit of sensitivity of the instrument and had to be smoothed by adjacent averaging for representation 


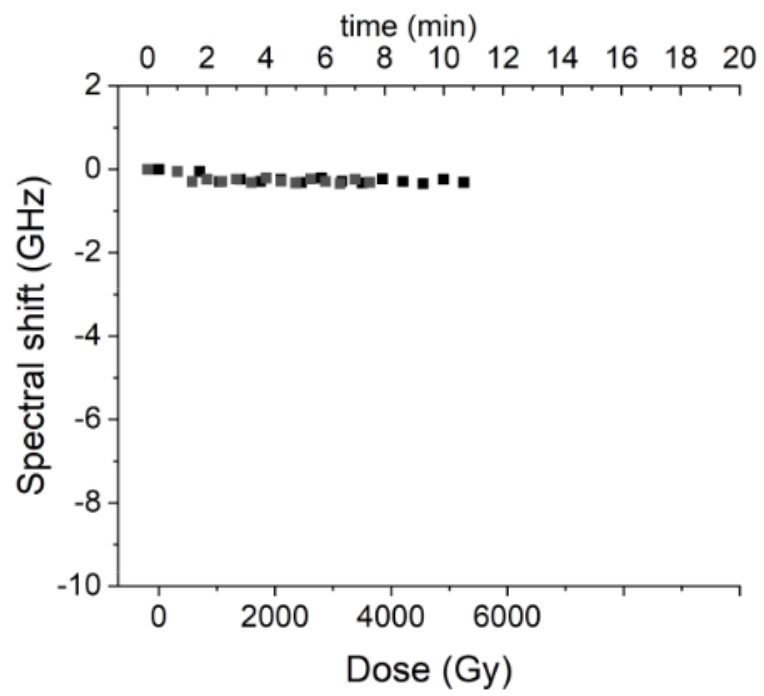

Fig. 7. Evolution of the spectral shift for an SMF exposed at $700 \mathrm{~Gy} / \mathrm{min}$.

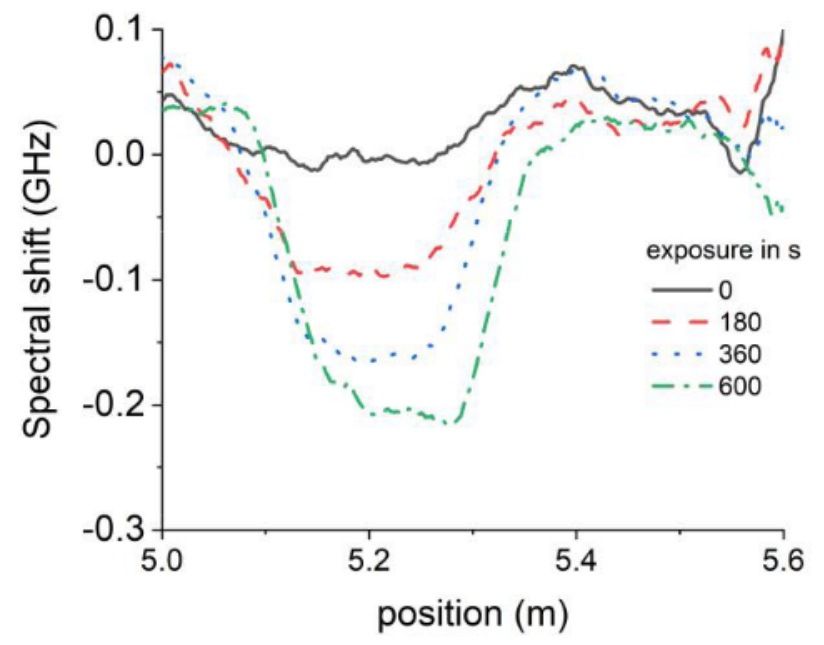

Fig. 8. Spectral shift versus position for the EBF at different times of exposure at $22 \mathrm{~Gy} / \mathrm{min}$.

in Figs. 8 and 9. Therefore, the actual relationship between spectral shift and dose for the considered fiber will have to be further investigated by repeated measurements.

In the third set of experiments, the Al-doped fiber was also tested at an even lower dose rate of $2 \mathrm{~Gy} / \mathrm{min}$, considering that a total dose of $2 \mathrm{~Gy}$ is a reference value for a single session of conventional radiotherapy treatment. The interesting outcome of these measurements is summarized in Figs. 10 and 11.

The first observation is that the spectral shift, and, thus, the correspondent refractive index change, has an opposite sign with respect to the case of higher doses. This confirms a qualitative observation that was also made during high-dose exposures, that is, the spectral shift of the irradiated region of the fiber seemed to grow and then quickly drops to negative values as the dose accumulates (notice that the data recording rate of one profile/30 s, limited by the instrument's settings of gauge resolution and measurement length, does not allow to clearly report the phenomenon on the graphs).

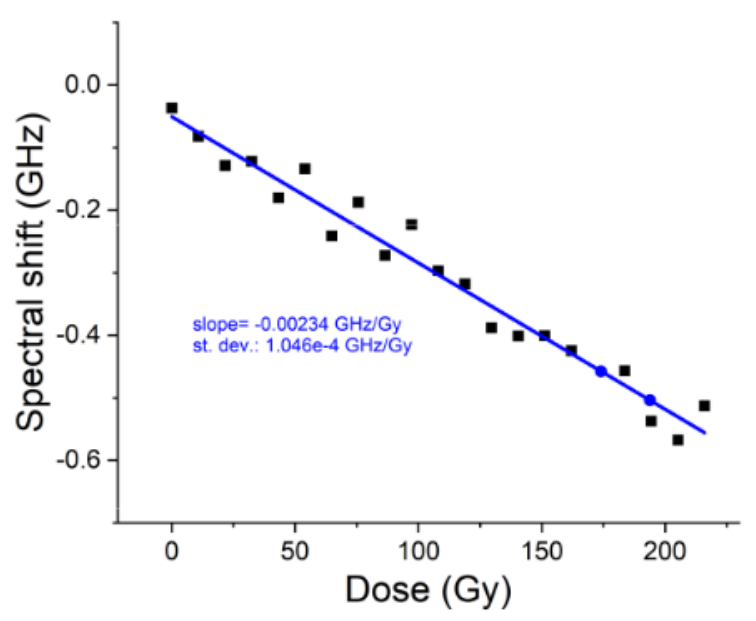

Fig. 9. Spectral shift versus accumulated dose at $22 \mathrm{~Gy} / \mathrm{min}$ for the EBF.

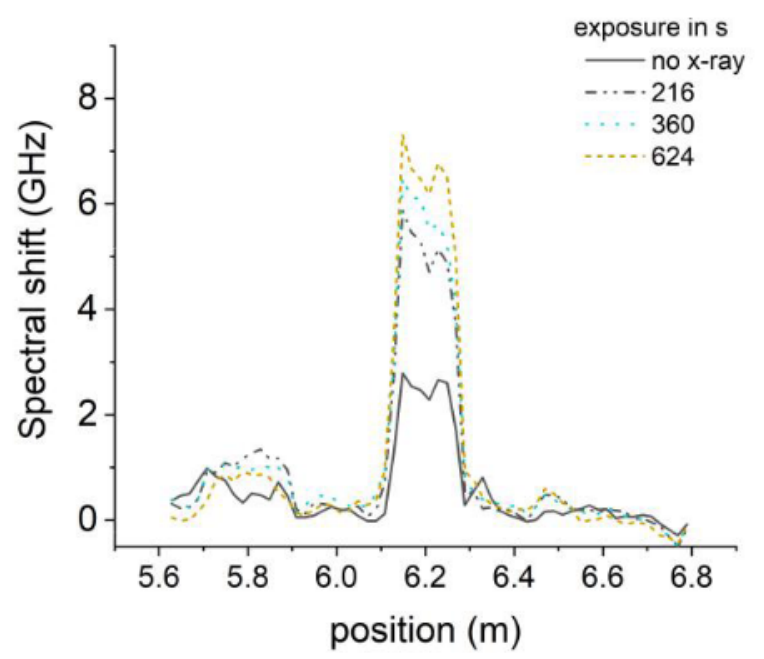

Fig. 10. Spectral shift versus position for the Al-doped fiber at different times of exposure at $2 \mathrm{~Gy} / \mathrm{min}$.

This suggests that there are different competing mechanisms of radiation-induced defects, one prevailing for low doses, but then quickly overcome as the dose increases. Fig. 11 shows a detectable growth with a nonlinear trend of the spectral shift and no bleaching effect. Instead, when the X-ray exposure is turned off, a smooth growth seems to occur, but this is likely to be an artifact since all the data are within the measurement noise as evident from the error bars. The height of the error bars is calculated as the standard deviation of the noise floor of all collected spectra shift spatial distributions (notice that Fig. 10 only reports few of the collected curves). The error bars are here reported to highlight that the low-dose measurement is affected by nonnegligible noise fluctuations that may also be ascribed to the close position of the OBR instrument to the radiation chamber.

Finally, the previous graphs demonstrate, as a proof-ofconcept, that the aluminosilicate fibers can be used as a distributed dosimeter by measuring the radiation-induced spectral shift with an OBR. Although the idea of using an EBF and an OBR for distributed sensing in therapeutic applications has previously been conceived [17] for measurement of the 


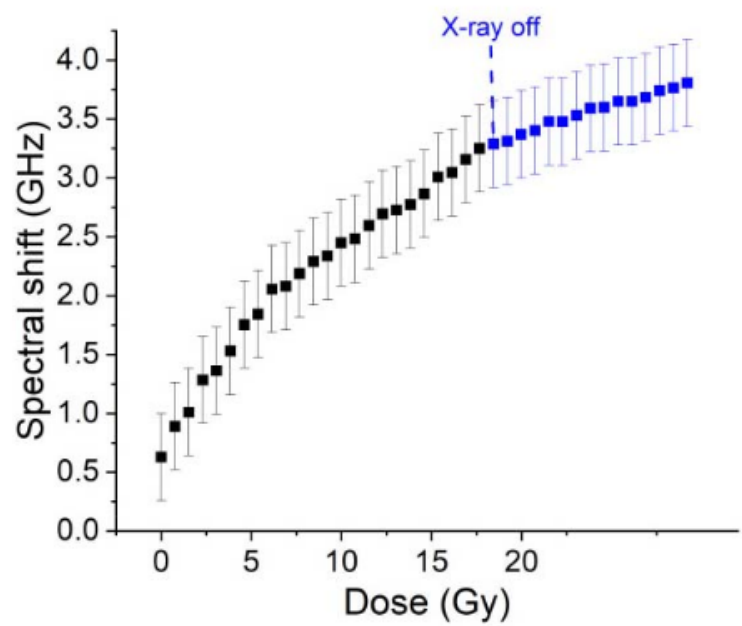

Fig. 11. Spectral shift versus accumulated dose at $2 \mathrm{~Gy} / \mathrm{min}$.

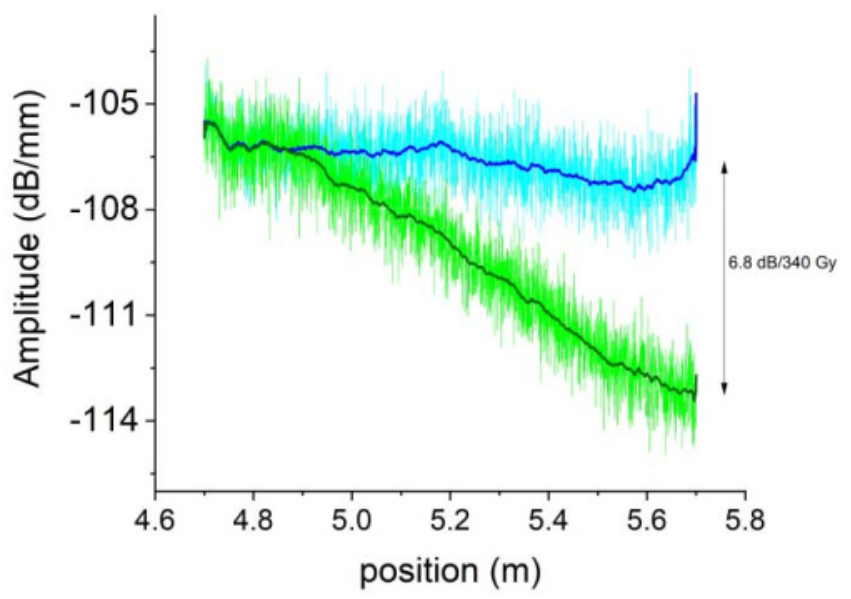

Fig. 12. Rayleigh backscattering signatures of a 1-m-long coiled fiber, as pristine (cyan curve) and after exposure at $22 \mathrm{~Gy} / \mathrm{min}$. An RIA of $6: 8 \mathrm{~dB}$ can be observed.

spatial distribution of temperature, this work represents the first demonstration of real-time in situ monitoring of radiation with an EBF and OBR technique.

The previous graphs also highlight that the spectral shift is a relevant parameter to track the dose evolution in time. On the other hand, a fairly simple measurement of RIA, which could be performed with a cost-effective OTDR, has been proved not to be effective. Indeed, during the previous experiments, the RIA was also measured by the OBR, but negligible values were recorded. In particular, in order to verify whether a weak RIA occurred, a 1-m-long coiled fiber was exposed at $22 \mathrm{~Gy} / \mathrm{min}$ for $10 \mathrm{~min}$. Fig. 12 reports the outcome of this test and shows an RIA of $6.8 \mathrm{~dB}$, meaning about $0.07 \mathrm{~dB}$ over $10 \mathrm{~mm}$. Such a low value is close to the amplitude resolution limit of an OTDR $(0.01 \mathrm{~dB})$. Together with its limited spatial resolution (about $10 \mathrm{~mm}$ ), OTDR seems, therefore, not as suitable as OBR for optical fiber dosimetry.

Further investigations were then performed in order to assess the potential use of the OBR/EBF system in $\mathrm{X}$-ray dosimetry. From an application viewpoint, it must

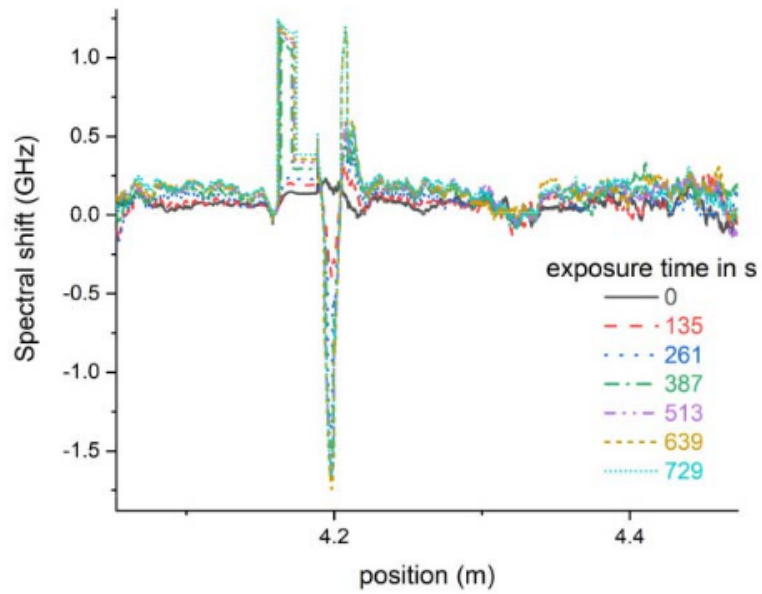

Fig. 13. Spectral shift versus position for the Mg nanoparticle-doped fiber (NPEBF) exposed at $710 \mathrm{~Gy} / \mathrm{min}$.

be pointed out that conventional radiotherapy treatments are performed with a dose of about 2 Gy/day on a five-day treatment, and the standard dose rate is about $0: 2 \mathrm{~Gy} / \mathrm{min}$. With these values, the OBR/EBF system is borderline in terms of sensitivity. Therefore, new fibers with further enhanced backscattering capabilities will have to be developed to be employed for such applications. On the other hand, new radio-therapeutic techniques, such as flash radiotherapy (Flash-RT) and stereotactic body radiotherapy (SBRT), exploit much higher dose rates, hence matching the sensitivity of the proposed system [18]-[20], which, however, requires certain measurement cares to provide meaningful results. Therefore, the possibility to use more consolidated optical sensing approaches, such as those based on FBGs, would certainly help the wide deployment of these new fiber-based dosimeters. Besides, such an approach allows also overcoming the acquisition rate limitations intrinsic to the OBR approach, thus making the measure "more real-time."

Following these considerations, the experimental research was then focused on two tasks: 1) explore the possibility of tailoring the characteristics of the sensing fiber by proper doping of the core to enhance the sensitivity and 2) make a real-time comparison between the OBR/EBF that works as a fully distributed sensing system and a conventional optical fiber quasi-distributed measurement, realized with an array of FBGs interrogated with a standard high-speed FBG interrogator.

\section{B. Nanoparticle-Doped EBF}

The first task was implemented by testing the magnesium-based $(\mathrm{Mg})$ nanoparticle-doped EBF (NPEBF) at high and low doses, as reported in Figs. 13-16. A straightforward comparison with the results of the Al-doped fiber (EBF) (see Figs. 10 and 11) highlights a lower sensitivity, especially at high doses, but the same behavior (i.e., positive spectral shift at a low dose and negative spectral shift at high dose) is observed. Unfortunately, this first NPEBF has so far exhibited lower detection capabilities, 


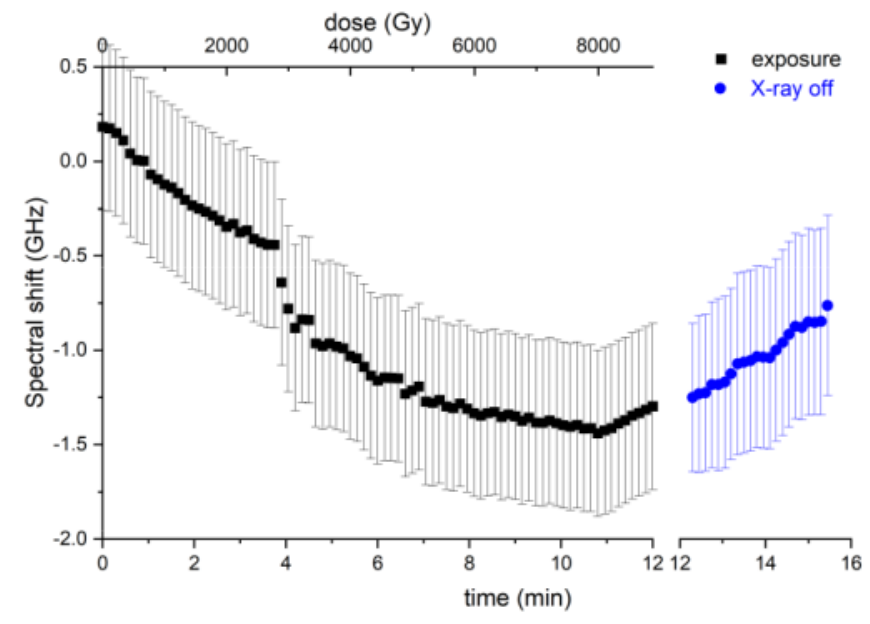

Fig. 14. Evolution of the spectral shift peak of the NPEBF fiber exposed at $710 \mathrm{~Gy} / \mathrm{min}$. The break of the horizontal axis is an artifact for more convenient reading.

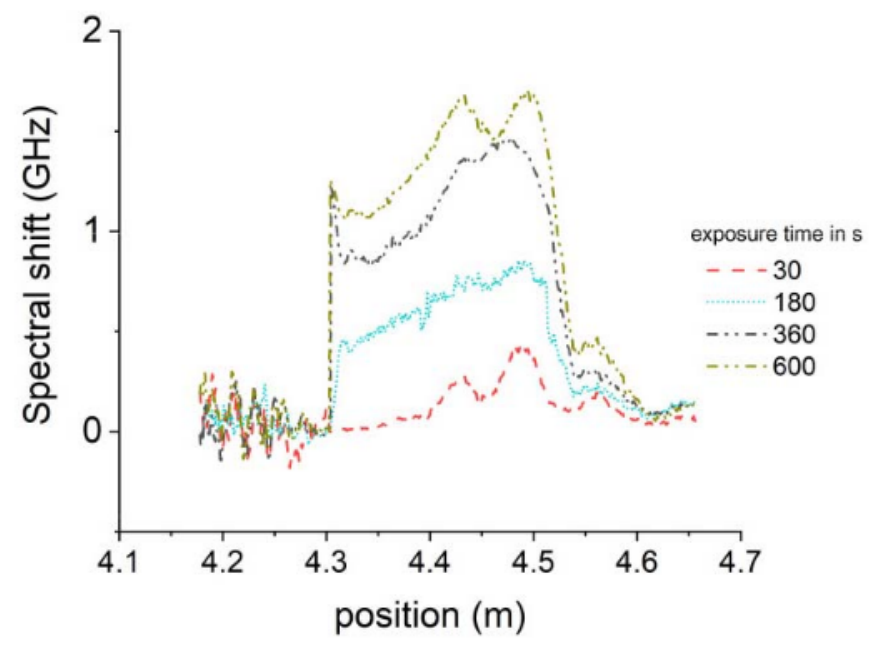

Fig. 15. Spectral shift along the NPEBF exposed at the therapeutic dose of $2: 2 \mathrm{~Gy} / \mathrm{min}$. It can be observed a positive trend, opposite to that observed at high doses (see Fig. 13), just as it occurs for the Al-doped fiber.

but it is expected that further engineering on the core doping (nanoparticles size and concentration) can improve its performance.

\section{Quasi-Distributed FBG Sensor}

For this experiment, a quasi-distributed sensor was fabricated in the NPEBF by writing a dense array of FBGs with a femtosecond laser using a technique similar to that described in [21] for SMFs. The FBGs had a length of $5 \mathrm{~mm}$ each, spaced by $2 \mathrm{~mm}$. The response of the FBG array was recorded by a commercial high-speed FBG interrogator (MicronOptics, model si255). Fig. 17 shows the spectral response of the FBG array. Unfortunately, the peak of the FBGs at 1541 and $1547 \mathrm{~nm}$ could be hardily detected by the peak tracking algorithm during the exposure experiments because of their lower reflectivity, and, therefore, had to be excluded from the analysis for too many artifacts. It is expected that the

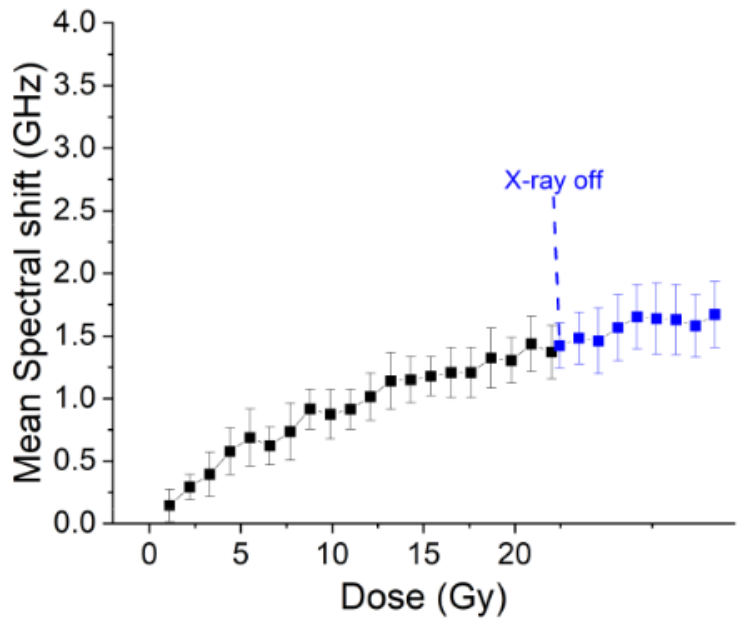

Fig. 16. Evolution of the spectral shift peak of the NPEBF exposed at $2: 2 \mathrm{~Gy} / \mathrm{min}$

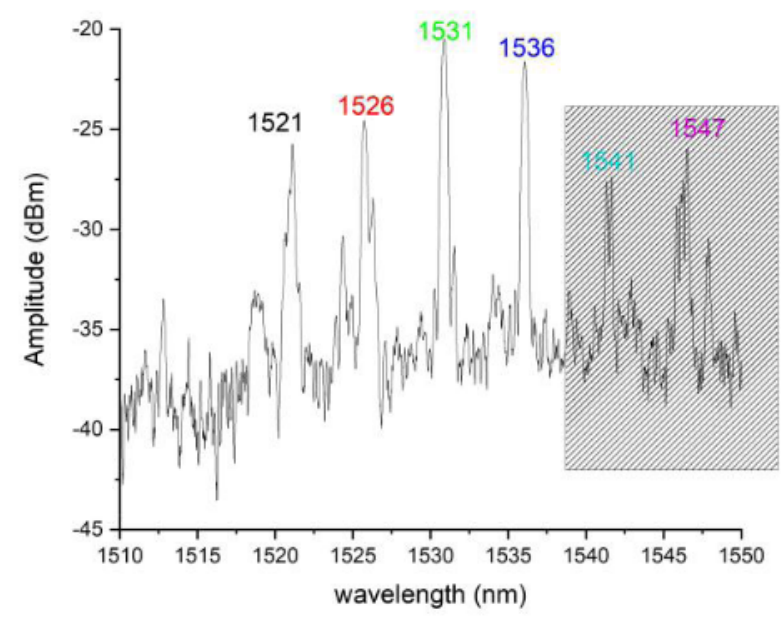

Fig. 17. Spectral response of the FBGs inscribed into the NPEBF by femtosecond direct laser writing. The FBGs at 1541 and $1547 \mathrm{~nm}$ (in the gray-pattern frame) could not be tracked during the irradiation experiment.

quality of the femtowritten FBGs will improve as the NPEBF is optimized.

The real-time comparison between distributed and quasidistributed sensings was made by exposing during the highdose experiment of the NPEBF fiber a twin sample with the FBG array. The Bragg wavelengths were recorded at $1 \mathrm{kHz}$, and the data were smoothed by an adjacent averaging of 1000 samples. Fig. 18 depicts the evolution of the Bragg peaks during exposure at $710 \mathrm{~Gy} / \mathrm{min}$. The gratings exhibit an absolute shift up to about $33 \mathrm{pm}$, which corresponds to an RIRIC in the order of $3 \cdot 10^{5}$. Notice that both red and blue shifts of the Bragg peaks are observed, which further supports the hypothesis of two competing phenomena in the radiation-induced defects as it is observed when recording the spectral shift with the OBR. As expected, the acquisition rate with the FBG-based approach can be set much higher than that with the OBR/EBF system (kilohertz versus hertz), but this occurs at the price of a reduced resolution and/or limited 


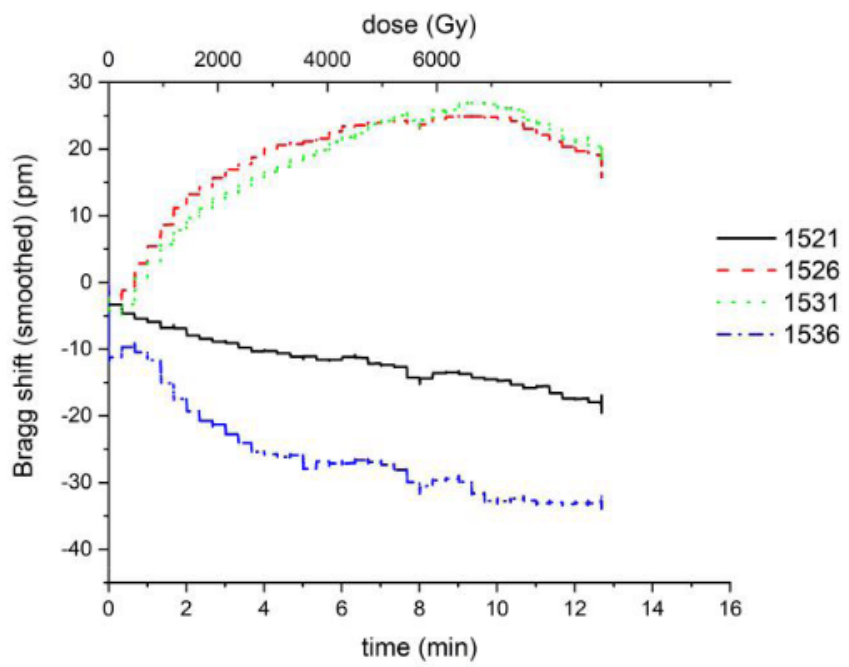

Fig. 18. Bragg shift of the FBGs inscribed into the NPEBF and exposed at $710 \mathrm{~Gy} / \mathrm{min}$, during the same experiment of Figs. 13 and 14.

spatial range. Furthermore, an optical fiber requires an extra fabrication step to be equipped with an FBG array, which may be inconvenient for disposable dosimetry sensors. These pros and cons shall be then further investigated in a real framework.

\section{CONCLUSION}

A new setup for real-time distributed X-ray dosimetry based on fiber optics has been presented. The system exploits an $\mathrm{EBF}$ as the sensing element, interrogated with an OBR that can measure the local spectral shift of the Rayleigh scattering along with the fiber. The measurements demonstrate a detection capability down to $2 \mathrm{~Gy} / \mathrm{min}$ with a submillimeter resolution. The EBF is based on an ad hoc aluminum-doped fiber. Further experiments have been carried out using a magnesium nanoparticle-doped EBF in a preliminary version. The fibers were exposed from high- $(700 \mathrm{~Gy} / \mathrm{min})$ to low-(2 Gy/min) dose rates, demonstrating that both types of fiber can be used as sensors, with the aluminum-doped fiber, exhibiting a higher sensitivity. The measurements also highlighted that there are two competing phenomena that induce the response to radiation, which shall be further investigated for the development of more sensitive optical fibers. The experimental research also highlighted that the OBR is likely the most suitable instrument to implement distributed dosimetry with optical fibers because: 1) along with the RIA, it can measure the RIRIC, which is a more sensitive parameter to radiation and 2) it performs measurements with high spatial resolution. A proof-of-concept comparison with a quasidistributed sensing system based on an array of FBGs was also performed, confirming the results and discussing the pros and cons of the two optical fiber-based sensing techniques. The overall work demonstrates that real-time dosimetry can be implemented in conventional and advanced radiotherapy treatments with the proposed system. Further developments shall be focused on extensive measurements to investigate new optical fibers with possible further enhanced sensitivity, a deep investigation of the physical effects of radiation on these fibers, and optimization of the instrument settings according to the application requirements (sensitivity, spatial resolution, and sampling rate). Last but not least, economical aspects (such as the use of the fiber sensor as a disposable probe and the cost of the backscattering reflectometer) must be carefully considered when moving from proof-of-concept research to biomedical applications. As a perspective, the system shall be tested in a real or simulated framework of radiotherapy treatment to better asset its advantages and limits.

\section{REFERENCES}

[1] A. Micke, D. F. Lewis, and X. Yu, "Multichannel film dosimetry with nonuniformity correction," Med. Phys., vol. 38, no. 5, pp. 2523-2534, May 2011.

[2] K. Pushpavanam et al., "Determination of topographical radiation dose profiles using gel nanosensors," Sci. Adv, vol. 5, no. 11, Nov. 2019, Art. no. eaaw8704.

[3] A. L. Palmer et al., "Comparison of methods for the measurement of radiation dose distributions in high dose rate (HDR) brachytherapy: Gedoped optical fiber, EBT3 gafchromic film, and PRESAGE radiochromic plastic," Med. Phys., vol. 40, no. 6, May 2013, Art. no. 061707.

[4] M. A. S. Zaghloul et al., "High spatial resolution radiation detection using distributed fiber sensing technique," IEEE Trans. Nucl. Sci. vol. 64, no. 9, pp. 2569-2577, Sep. 2017.

[5] D. D. Francesca et al., "Qualification and calibration of single-mode phosphosilicate optical fiber for dosimetry at CERN," J. Lightw. Technol., vol. 37, no. 18, pp. 4643-4649, Sep. 15, 2019.

[6] Z. Ding et al., "Distributed optical fiber sensors based on optical frequency domain reflectometry: A review," Sensors, vol. 18, no. 4, p. 1072 , Apr. 2018.

[7] M. Olivero et al., "Preliminary investigation of radiation dose sensors based on aluminum-doped silicate optical fibers," in Proc. IEEE Int. Symp. Med. Meas. Appl. (MeMeA), Jun. 2020, pp. 1-5.

[8] OBR4600 Manual, Chapt. 8. Luna Inc. Roanoke, VA, USA Accessed: Mar. 9, 2020. [Online]. Available: https://lunainc.com/ product/sensing-solutions/obr-4600/

[9] B. J. Soller, D. K. Gifford, M. S. Wolfe, and M. E. Froggatt, "High resolution optical frequency domain reflectometry for characterization of components and assemblies," Opt. Exp., vol. 13, no. 2, pp. 666-674, 2005.

[10] M. E. Froggatt and J. Moore, "High-spatial-resolution distributed strain measurement in optical fiber with Rayleigh scatter," Appl. Opt., vol. 37, no. 10 , pp. $1735-1740,1998$.

[11] L. Cognolato, "Chemical vapour deposition for optical fibre technology," Le J. de Phys. IV, vol. 05, no. C5, pp. C5-975-C5-987, Jun. 1995, doi: 10.1051/jphyscol: 19955115

[12] J. E. Townsend, S. B. Poole, and D. N. Payne, "Solution-doping technique for fabrication of rare-earth-doped optial fibres," Electron. Lett., vol. 23, no. 7, pp. 329-331, Mar. 1987, doi: 10.1049/el:19870244.

[13] W. Blanc et al., "Compositional changes at the early stages of nanoparticles growth in glasses," J. Phys. Chem. C, vol. 123, no. 47, pp. 29008-29014, Nov. 2019.

[14] W. Blanc et al., "Fabrication of rare earth-doped transparent glass ceramic optical fibers by modified chemical vapor deposition," J. Amer. Ceram. Soc., vol. 94, no. 8, pp. 2315-2318, Aug. 2011.

[15] R. Kashyap, Fiber Bragg Gratings. New York, NY, USA: Academic, 1999.

[16] S. Girard et al., "Overview of radiation induced point defects in silicabased optical fibers," Rev. Phys., vol. 4, Nov. 2019, Art. no. 100032.

[17] A. Beisenova et al., "Multi-fiber distributed thermal profiling of minimally invasive ther-mal ablation with scattering-level multiplexing in MgO-doped fibers," Biomed. Opt. Exp., vol. 10, no. 3, pp. 1282-1296, 2019.

[18] J. D. Wilson, E. M. Hammond, G. S. Higgins, and K. Petersson, "Ultrahigh dose rate (FLASH) radiotherapy: Silver bullet or fool's gold?' Frontiers Oncol., vol. 9, p. 1563, Jan. 2020, doi: 10.3389/fonc.2019.01563.

[19] K. J. Harrington, "Ultrahigh dose-rate radiotherapy: Next steps for FLASH-RT," Clin. Cancer Res., vol. 25, no. 1, pp. 3-5, Jan. 2019.

[20] R. M. de Kruijff, "FLASH radiotherapy: Ultra-high dose rates to spare healthy tissue," Int. J. Radiat. Biol., vol. 96, no. 4, pp. 419-423, Apr. 2020.

[21] A. Beccaria et al., "Temperature monitoring of tumor hyperthermal treatments with optical fibers: Comparison of distributed and quasidistributed techniques," Opt. Fiber Technol., vol. 60, Dec. 2020, Art. no. 102340. 


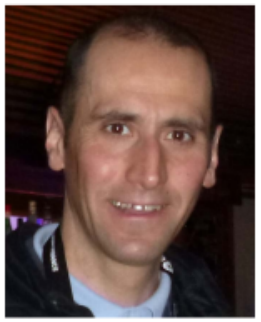

Massimo Olivero received the M.S. degree in electronic engineering and the $\mathrm{Ph} . \mathrm{D}$. degree in photonics from the Politecnico di Torino, Turin, Italy, in 2002 and 2006 , respectively.

He was a Visiting Researcher with the Technical University of Denmark, Kongens Lyngby, Denmark, from 2004 to 2005, where he worked on the development of integrated optical devices by direct UV writing. From 2006 to 2008, he was a Post-Doctoral Researcher with the Politecnico di Torino, where he conducted research on optical measurements and characterization of nanostructured materials. In 2010 and 2013, he was a Visiting Scientist with the Department of Physics, Universidade Federal de Pernambuco, Recife, Brazil, where he worked on femtosecond laser writing of waveguides and materials characterization by Optical Coherence Tomography. He is currently a Senior Technician with the Photonics Laboratory, Politecnico di Torino, where he mainly works on fiber components for sensing applications.

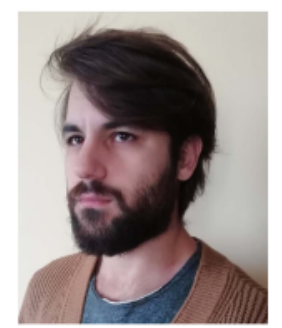

Alessandro Mirigaldi received the B.Sc. degree in physics engineering from the Politecnico di Torino, Turin, Italy, in 2016, and the joint M.Sc. degree in physics of complex systems from the Politecnico di Torino and Paris Diderot University, Paris, France, in 2018, where he is currently pursuing the Ph.D. degree in electronics, electrical and communications engineering.

His research interests include power scaling of high-power laser systems and fiber Bragg gratings manufacturing and application and optical fibers sensors.

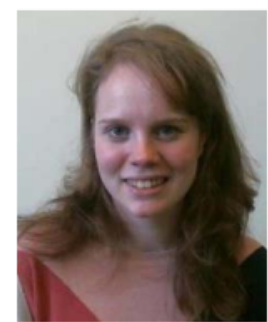

Valentina Serafini received the B.Sc. degree in biomedical engineering and the M.Sc. degree in electronic engineering from the Politecnico di Torino, Turin, Italy, in 2016 and 2019, respectively, where she is currently pursuing the Ph.D. degree in electronic engineering.

Her research interests include optical fiber sensors, biomedical sensors, and fiber lasers.

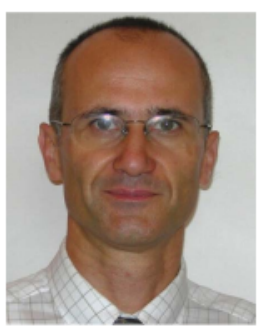

Alberto Vallan (Senior Member, IEEE) is currently an Associate Professor of electrical and electronic measurements with the Department of Electronics and Telecommunications, Politecnico di Torino, Turin, Italy. Since 2000, he has been a Lecturer in courses concerning electronic measurements and sensors. His research interests are focused on the development and characterization of fiber sensors and measuring instruments for biomedical and industrial applications.

Mr. Vallan is a Senior Member of the IEEE/I\&M Society.

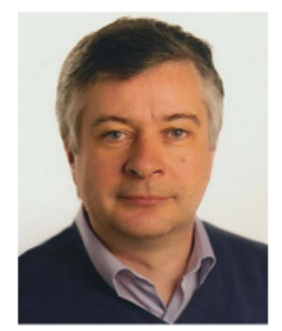

Guido Perrone (Member, IEEE) received the $\mathrm{Ph}$.D. degree in electromagnetics/photonics from the Politecnico di Torino, Turin, Italy, in 1994.

$\mathrm{He}$ is currently a Professor of optical fibers and components with the Politecnico di Torino. He has authored or coauthored the book "Fiber-Optic Sensors for Biomedical Applications" (Artech House) and more than 200 publications in international journals and conferences. His research activity is mainly in the fields of fiber optic sensors and of high power lasers for industrial and biomedical applications.

Dr. Perrone is a Senior Member of the Optical Society of America and a member of the IEEE/MTTS and IEEE/Photonics Society.

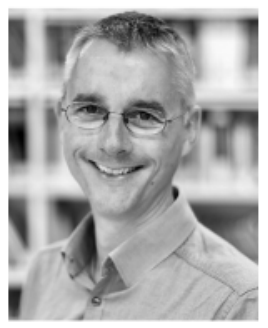

Wilfried Blanc received the M.Sc. and Ph.D. degrees in physics from University Claude Bernard, Villeurbanne, France, in 1996 and 2000 respectively.

He held a post-doctoral position with the ICMCB Laboratory, University of Bordeaux, Bordeaux, France, funded by Rhodia-Solvay. In 2002, he commenced with the Centre National de la Recherche Scientifique (CNRS), Laboratoire de Physique de la Matière Condensée (now Institut de Physique de Nice), Nice, France, where his main interests are the design, realization and characterization of rare-earth-and nanoparticles-doped silica optical fibers which are made by using modified chemical vapor deposition (MCVD) technique.

Mourad Benabdesselam received the M.Sc. and Ph.D. degrees in electronics from Centre d'Electronique de Montpellier, Montpellier, France, in 1988 and 1991, respectively.

$\mathrm{He}$ is currently a Full Professor of electronics and electrical engineering with University Côte d'Azur, Nice, France. He is a member of the group Fibres Optiques et Applications, Institut de Physique de Nice (INPHYNI) UMR CNRS 7010, Nice. His main research interests concern the design and the characterization of optical fibers for the radiative environment and dosimetry.

Franck Mady received the Ph.D. degree in materials physics in 2002.

He has been an Associate Professor of Physics with Université Côte d'Azur (UCA), Nice, France, since 2003. He was the Head of the Professional Degree in Medical Dosimetry, UCA, from 2010 to 2018. He is currently the CoHead of the Fédération Claude Lalanne, a unique transdisciplinary research federation merging the various UCA expertises in mathematics, physics, image processing, AI, biology, and medicine for advancing radiation therapy practices. His research interests include the transport of radiation-induced carriers and space charge effects in insulating, and the study of radiationinduced mechanisms in silica optical fibers materials with applications in radiation hardening and sensing.

Carlo Molardi (Member, IEEE) received the master's degree in telecommunication engineering, working on numerical methods for optics and electromagnetism, and the Ph.D. degree in information technologies from the University of Parma, Parma, Italy, in 2011 and 2016, respectively.

$\mathrm{He}$ is currently an Assistant Professor with the School of Engineering and Digital Sciences, Nazarbayev University, Nur-Sultan, Kazakhstan. He previously covered a post-doctoral position with the Nazarbayev University and a post-doctoral position with the Information Engineering Department, University of Parma. His research interests include fiber optics, fiber sensors, photonic crystal fibers design, fiber lasers for high power operations, random lasers, and numerical methods for optics and electro-magnetism

Daniele Tosi received the B.Sc. and M.Sc. degrees in telecommunication engineering and the Ph.D. degree in electrical and computer engineering from the Politecnico di Torino, Turin, Italy, in 2004, 2006, and 2010, respectively.

$\mathrm{He}$ is an Associate Professor of electrical and computer egineering with Nazarbayev University, Nur-Sultan, Kazakhstan, where he is the Head of the Biosensors and Bioinstruments Laboratory, National Laboratory Astana. His research interests include optical fiber sensors, biomedical sensors, distributed sensing, and biosensors.

Dr. Tosi was a recipient of the IEEE Sensors Council Early Career Technical Award in 2018. He is an Associate Editor of the IEEE SENSORS JOURNAL. 